\title{
Soziogeographische Variation im Deutschen und \\ ihre Übersetzung ins Italienische
}

\author{
Lucia Cinato (Turin)
}

\begin{abstract}
The translation of multilingual texts, in which diatopic, diastratic and diaphasic varieties contribute to the characterization of the figures and their milieus, poses the problem of the sociolinguistic adequacy of the translation. It is well known that the greatest challenge lies in the varieties caused by the spatial variation of the language, since it is practically impossible to establish perfect equivalence between dialects. However, the reproduction of diamesic varieties, in particular the written reproduction of the spoken language, can cause great difficulties for translators. The article presents the TRADIVARIO project, the aim of which is to investigate strategies and tendencies in the translation of multilingual literary texts for the GermanItalian language pair that are characterized by a pronounced sociolinguistic stratification or representative of the variety structure of the spoken language of the present. In addition, the concrete goal of the project is the creation of a parallel corpus as well as the development of a digital platform, which makes the results of the investigation researchable and which can serve as a tool both for translators and learners and as an empirical basis for contrastive linguistic research.
\end{abstract}

\section{$1 \quad$ Einleitung1}

In diesem Beitrag wird das Forschungsprojekt TRADIVARIO, „Soziogeographische Variation und Übersetzung: Strategien und Tendenzen beim Sprachpaar Deutsch-Italienisch“, eine Zusammenarbeit innerhalb des Instituts für Germanistik der Universität Turin ${ }^{2}$ im Kontext der Digital Humanities, vorgestellt. Ziel des Projektes ist es, Strategien und Tendenzen bei der Übersetzung belletristischer Texte zu untersuchen, die durch eine ausgeprägte soziolinguistische Schichtung gekennzeichnet und für das Varietätengefüge der deutschen Gegenwartssprache repräsentativ sind. Bei der Übersetzung von Texten, in denen diatopische, diastratische und diaphasische Varietäten zur Charakterisierung der Figuren und deren Milieus eine Rolle spielen, stellt sich das Problem der soziolinguistischen Adäquatheit der Übersetzung. Wie an verschiedenen Stellen in der übersetzungswissenschaftlichen Literatur nachgewiesen (cf. Albrecht 2005; Berruto 2010; Schreiber 2007), stellen die Varietäten, die durch die räumliche Variation bedingt sind, die größte Herausforderung dar - da es kaum möglich scheint, eine Äquivalenz

\footnotetext{
${ }^{1}$ Zu diesem Thema cf. auch Cinato/Amico di Meane (2019).

2 Das Projekt wird von Marcella Costa und mir durchgeführt. Wir bedanken uns bei Isabella Amico di Meane für die Teilnahme an der ersten Phase des Projektes.
} 
zwischen Dialekten verschiedener Ausgangs- und Zielsprachen herzustellen. Aber auch die Wiedergabe diamesischer Varietäten, insbesondere die schriftliche Wiedergabe des Gesprochenen (,fingierte Mündlichkeit“" nach Goetsch 1985), kann im Übersetzungsprozess große Schwierigkeiten bereiten (cf. Nicklaus 2015). Nach einer kurzen Vorstellung des Forschungsprojektes werden hier die ersten Ergebnisse einer Pilotstudie über einen für den Corpus ausgewählten Roman, Happy Birthday, Türke! von Jakob Arjouni (1985) mit seiner Übersetzung durch Maneri (1993), erläutert.

\section{Das Projekt}

Die Relevanz eines Projektes, das sich ausführlich mit der Übersetzung sozio-geographischer Varietäten - mit besonderem Fokus auf der Wiedergabe des Gesprochenen im Geschriebenen befasst -, entsteht aus verschiedenen Überlegungen; hierzu zählen u. a. objektive Schwierigkeiten bei der Übersetzung von soziolinguistisch geprägten Texten (insbesondere auf der diamesischen Ebene), eine geringe Aufmerksamkeit für diese Phänomene im übersetzungs- und sprachwissenschaftlichen Bereich, ein Mangel an systematischen Studien über dieses Thema für das Sprachenpaar Deutsch-Italienisch, ${ }^{3}$ das Bedürfnis nach didaktischen Materialien für Übersetzungsseminare in Masterstudiengängen und nicht zuletzt die heutige Tendenz im literarischen Bereich zu einer größeren Permeabilität zwischen Mündlichkeit und Schriftlichkeit als Folge des Einflusses vom Gesprochenen auf das Geschriebene (cf. Calaresu 2005: 65f.; Schwitalla/Tiittula 2009: 30-31). Letzteres ist kennzeichnend für eine Vielzahl literarischer Produktionen in der Originalsprache, i. e. es entstehen immer mehr Werke, die das Varietätengefüge der gesprochenen Sprache, literarische Stilisierungen des realitätsnahen Gesprochenen, intralinguistische Variationen und sprachliche Anpassung sowohl der Protagonisten als auch der Autorinnen und Autoren aufweisen (cf. Assman/Menzel 2018; Betten 1994). In diesem Zusammenhang müssen auch die veränderte Sensibilität der Leser für die Sprachvariation und die unterschiedliche Wahrnehmung bestimmter Besonderheiten im Text gesehen werden. Diese sind einerseits mit der fortschreitenden Nivellierung von den im öffentlichen Diskurs typischen Mitteln der öffentlichen Sprache und den spontanen, umgangssprachlichen Formen der privaten Sprache verbunden und andererseits mit einer Sprache, die in der heutigen globalisierten Gesellschaft einen zunehmend Variationsreichtum zeigt. Daher das Bedürfnis nach Übersetzungen, die die Vielfalt und die sprachliche sowie stilistische Komplexität der Originaltexte wiedergeben können.

Das Projekt sieht die Erstellung eines recherchierbaren Parallelkorpus sowie die Entwicklung einer digitalen Plattform vor, auf der die Ergebnisse der Untersuchung gesammelt und nach Übersetzungsrichtung und sozio-geographischer Varietät - als Archiv der guten Übersetzungspraktiken - systematisiert werden. Die Ergebnisse der Untersuchung sollen als Hilfsinstrument

\footnotetext{
${ }^{3}$ Die Übersetzung von sozio-geographischen, besonders diaphasischen Varietäten, ist in übersetzungswissenschaftlichen Überlegungen ein häufig behandeltes Thema, man denke z. B. an die vielen Studien über die Übersetzungen von Camilleri. Der Untersuchungsgegenstand des beschriebenen Projektes ist aber eine Systematisierung der Variationsphänomene und deren Übersetzung.
} 
sowohl professionellen Übersetzern als auch Lernenden dienlich sein - und ebenso als empirische Basis für theoretisch-deskriptive und angewandte kontrastive Untersuchungen. ${ }^{4}$

\section{$3 \quad$ Stand der Forschung}

Angesichts der manchmal starken sprachlichen Nachahmung in der zeitgenössischen Literatursprache (man denke nur an Kanak Sprak von Feridun Zaimoglu 1995) fällt es oft schwer, im Zieltext adäquate Lösungen zu finden, die in der Lage sind, die soziolinguistische Vielfalt des Originals wiederzugeben. Die Gründe dafür sind vielfältig, z. B. Asymmetrien zwischen den Varietäten in der Ausgangs- und in der Zielsprache, der größere Konservatismus, der den Übersetzungen innewohnt (cf. Toury 1995: 2725), eine absichtliche Anhebung des Registers. Symptomatisch für eine Veränderung der Sensibilität und ein größeres Bewusstsein für das Problem der Darstellung des Substandards sind die jüngsten Neuübersetzungen von Klassikern, die darauf abzielen, die sprachlichen Eigenheiten von Texten wiederherzustellen, in denen die soziogeografische Variation eine Schlüsselrolle spielt. So sind zum Beispiel die neuen Übersetzungen von Kleiner Mann - was nun? von Fallada (1933/2008) und der Blechtrommel von Grass (2009) im Vergleich zu den ersten Übersetzungen, die von Nivellierungen und Kompensationsstrategien geprägt sind, dem Originaltext treuer, weil sie die stilistischen Merkmale des Quelltextes und die Substandard-Elemente deutlicher machen, wie im Folgenden Beispiel aus der Blechtrommel zu beobachten ist (cf. Costa 2015: 82-84, Hervorhebung L. C.):

(1) $[\ldots]$,Is sowieso nich mein Typ“, winkte Herbert ab. „Nu sieh dich die Speckfalten an und das Doppelkinn, was sie hat.“ Herbert hielt den Kopf schief und machte sich Vorstellungen: N2a und das Kreuz, wie'n Zweimännerspind. Häbert is mehr für zierliche Damen, so klaine Luderchen wie Püppkens.“(Grass 1959/2007: 187f.)

[...] "Tanto, non è il mio tipo," disse Herbert. "Guardatela un po', grassa com'è, e col doppio mento". La osservava tenendo la testa un po' china da un lato: "E poi guarda le reni, sono come uno stipetto per due persone. Herbert è per le donnine, per quelle puttanelle che paiono bambolotte." (Grass/Secci 1962/1989: 185f.)

[...] "Tanto non è mica il mio tipo," declinava Herbert. "Guarda lì quelle pieghe di ciccia, e quel po' po' di doppiomento che tiene." Herbert inclinava la testa da un lato e fantasticava: "Vabbe' e quel didietro, che pare un armadio per due. Herbert è più per le signore delicate, le puttanelle piccoline tipo bambole". [...] (Grass/Bianchi 2009: 190-192)

Das Beispiel zeigt, dass beide Übersetzungen die phonetisch-graphematischen Besonderheiten, die in der Übersetzung kaum übertragbar sind, vernachlässigen. Es werden aber einige Versuche unternommen, die syntaktische Ebene zu reproduzieren (Rhematisierungen, segmentierte Sätze etc.). Die meisten Versuche, den informellen umgangssprachlichen Ton wiederzugeben, lassen sich aber auf lexikalische Ebene konstatieren, wenn auch mit unterschiedlichen Ergebnissen: Die neuere Übersetzung von Grass/Bianchi entscheidet sich für einen Stil, der eher auf

\footnotetext{
${ }^{4}$ Leider wurde das Projekt, dass zuerst als PRIN-Projekt entstanden ist, nicht finanziert, weshalb die digitale Plattform noch nicht realisiert werden konnte.

5 Siehe dazu auch die Debatte über die Übersetzungsuniversalien (translation universals) in Baker (1996) und die Beiträge auf italienischer Seite von Garzone/Cardinaletti (2004), Cardinaletti/Garzone (2005) und Ondelli/Viale (2010).
} 
einem informellen Register basiert, das diatopisch und diastratisch markiert ist (cf. Costa 2015: 84).

Wie die Studie von Schwitalla/Tiittula 2009 bestätigt, nehmen Übersetzungen in dem Versuch, die Formen der Mündlichkeit zu reproduzieren, seltener die Bandbreite der sprachlichen und textlichen Werkzeuge wahr, die in der einheimischen Literatursprache verwendet werden. Mit anderen Worten: Phänomene, die in den Originaltexten für die Simulation der gesprochenen Sprache vorkommen, werden in den Zieltexten reduziert, wenn sie nicht sogar vollständig fehlen, und vorzugsweise auf lexikalischer und syntaktischer Ebene wiedergegeben. Neben der daraus resultierenden Verschiebung zugunsten der Standardsprache wird die Kohärenz der gesamten Übersetzung auch durch häufiges Register- und Styleshifting beeinträchtigt. Es wurden folgende allgemeinsprachliche Tendenzen in der Übersetzungspraxis festgestellt: Die Tendenz zur Standardisierung bzw. Einebnung der Sprachvariation (,law of growing standardization“, Toury 1995: 267-274 ); die Tendenz zur Verlagerung der diatopischen Markierung, die nach Berruto 2010 grundsätzlich unübertragbar ist, auf die diaphasische und diastratische Dimension (Albrecht 2005); sowie die Tendenz zur Reduzierung der für die Nachahmung des Gesprochenen funktionalen Phänomene bzw. deren Konzentration auf die lexikalische Ebene (cf. Schreiber 2007; Schwitalla/Tiittula 2009). Unsere Aufgabe ist es daher zu erforschen, ob diese Tendenzen auch für das Sprachenpaar Deutsch-Italienisch bestätigt werden.

\section{Korpus und Vorgehen}

Das Korpus besteht aus belletristischen Texten verschiedener Gattungen (Romane, Graphic Novels, Biographien etc.), die nach dem Kriterium der Repräsentativität für das Varietätenspektrum des Gegenwartsdeutschen ausgewählt wurden. Hier eine Auswahl der zu untersuchenden Texte und deren Übersetzungen:

Arjouni (1985/2003): Happy Birthday, Türke! (Übersetzung von Maneri 1993/2009)

Jalowicz-Simon (2014): Untergetaucht. Eine junge Frau überlebt in Berlin 1940-1945 (Übersetzung von Amico di Meane 2015)

Kara (2003): Selam Berlin! (Übersetzung von Pugliano 2005)

Kleist (2012): Der Boxer (Übersetzung von Patrucco Becchi 2015)

Meyer (2006): Als wir träumten (Übersetzung von Gado-Cravero 2016)

Schulze (1998): Simple Storys (Übersetzung von Groff 2008)

Süskind (1984): Der Kontrabass (Übersetzung von Agabio 1986)

Zaimoğlu (1997): Abschaum. Die wahre Geschichte von Ertan Ongun (Übersetzung von Orsi 1999)

Die Analyse der jeweiligen Texte, die sich auf den theoretisch-deskriptiven Rahmen von Schwitalla/Tiittula (2009) stützt und hier am Beispiel von Jakob Arjounis Happy Birthday, Türke! vorgestellt wird, gliedert sich in zwei Schritte: Zuerst werden das Original und die Übersetzung separat betrachtet und jeweils die sprachlichen Variationsphänomene auf den verschiedenen Analyseebenen - phonetisch-graphematisch, semantisch, syntaktisch und lexikalisch klassifiziert. Besonderer Fokus liegt dabei auf den typisch dialogischen und umgangssprachlichen Formen, die sowohl der Autor als auch der Übersetzer benutzen, um die Illusion der Oralität zu erschaffen bzw. zu reproduzieren. Die erste Phase der Analyse zielt daher darauf ab, die 
Aspekte zu ermitteln, die „dem Geschriebenen helfen, so zu tun, als wäre es gesprochen“(Calaresu 2005: 68), z. B. Echtzeit-Planungssignale, Ellipsen und telegrafischer Stil (nominaler Stil), anaphorische Ketten mit wenig lexikalischer Vielfalt, Wiederholungen, deiktische Signale etc.

$\mathrm{Zu}$ einem späteren Zeitpunkt werden die Übersetzungsstrategien in Bezug auf die oben genannten Analyseparameter und die angewandten Strategien zur Wiedergabe der beobachteten Phänomene analysiert, unter besonderer Berücksichtigung eventueller Kompensationsstrategien zur Erhaltung soziolinguistischer Markierung. Ausgehend davon, dass stilistische und soziolinguistische Veränderungen nicht nur die die Charakterisierung der Figuren, sondern auch die Gesamtwirkung eines Werkes verändern, stellen wir uns die folgende Frage: Wie wird die soziolinguistische Markierung des Originals in der Übersetzung wiedergegeben? In differenzierter Weise durch die Verwendung von verschiedenen Indikatoren oder durch Stilisierung einer begrenzten Anzahl von Merkmalen und die Konzentration auf einer bestimmten Ebene der Sprachbeschreibung? Welche Kompensationsstrategien benutzt der Übersetzer und welche Folgen hat ihre Verwendung für den Zieltext? Nach dem von Toury (1995: 267-274) formulierten Gesetz der wachsenden Standardisierung (,,law of growing standardization“), neigen Übersetzungen dazu, soziolinguistische und stilistische Variationen des Originals zu reduzieren, was manchmal zu einer Nivellierung der Idiolekte der Charaktere führt. Spiegelt sich dieses allgemeine Merkmal der Übersetzung auch in den zu untersuchenden Übersetzungen wider?

\section{Soziolinguistische Variation in Happy Birthday, Türke von Jakob Arjouni}

Wir stellen im Folgenden eine synthetische Darstellung der Analyseergebnisse des ersten untersuchten Romans Happy Birthday, Türke von Jakob Arjouni und seiner italienischen Übersetzung durch Gina Maneri (1993/2009) vor.

Zuerst ein Überblick über die soziolinguistische Variation im Roman: Die Erzählung in der ersten Person ist dem Protagonisten selbst anvertraut, der es liebt, sich im Einklang mit seinem Temperament und seinem ungeregelten Lebensstil auf provokative Weise und mit einer guten Dosis Sarkasmus auszudrücken. Die Sprache, in der Kayankaya seine Geschichte erzählt, ist eine sogenannte standardnahe Umgangssprache, ohne dialektale Elemente, gekennzeichnet durch einige phonetische und lexikalische Reduktionen; die Sätze sind kurz, der Ablauf ist hauptsächlich parataktisch, der Wortschatz direkt und bunt. Diese nähesprachlichen Elemente werden mit distanzsprachlichen Merkmalen vermischt (größere Informationsdichte, Komplexität, Elaboriertheit, Planung etc.; cf. Koch/Oesterreicher 1985).

Türkische Immigranten, Prostituierte und Zuhälter, Drogenabhängige, Polizisten verschiedener Grade, Postbeamte gehören zum differenzierten Milieu Frankfurts, dem Arjouni in den Dialogen des Romans eine Stimme gibt. Jede dieser sozialen Schichten ist durch eine jeweils spezifische Ausdrucksweise gekennzeichnet. Kayankaya als Figur spricht jedoch in einer Sprache, die in vielerlei Hinsicht lässiger und „ungrammatischer“ ist als die des Kayankaya-Erzählers. Seine Sprache zeigt dennoch, dass er in der Lage ist, seine eigene Ausdrucksweise entsprechend der kommunikativen Situation und dem Gesprächspartner zu modulieren, i. e. von einem rein informellen Ton, manchmal sogar Slang, zu einem entschieden formellen Register überzugehen. 
Auf der diatopischen Ebene finden sich der Frankfurter Dialekt und das Ruhrdeutsch, auf der diastratischen Ebene Dealer- und Fixerjargon, Gaunersprache sowie Ausländerdeutsch. Auf der diaphasischen Ebene kommt schließlich eine breite Palette von Registern vor, die sich auf einem Kontinuum ansiedeln, das von einem Maximum an Formalität und sprachlicher Standardisierung über ein Maximum an Markiertheit in den verschiedenen Variationsdimensionen reicht - bis hin zum Dialekt.

Im Folgenden werden einige Belege vorgestellt, die zeigen, wie die standardnahe Umgangssprache im Roman simuliert wird. ${ }^{6}$ Auf der phonetisch-graphematischen Ebene ist diese Vielfalt durch zahlreiche Phänomene der phonetischen und lexikalischen Reduktion (was, grad, heut), Kontraktion und Enklisis (nicht immer durch das Apostroph angegeben: gibts, wirs) sowie die Reduktion des unbestimmten Artikels ('ne Gabel; 'ner Stunde) gekennzeichnet. Funktional für die Nachahmung des Gesprochenen sind auf der lexikalischen Ebene:

- die zahlreichen Modalpartikeln (eigentlich, denn, bloß);

- die primären (aha, ei) und sekundären (oh Gott, Mann) Interjektionen;

- Steigerungsmittel (verdammt, anständig, schön);

- Ausdrücke von Vagheit (so was, und alles);

- die Verwendung des Demonstrativpronomens anstelle des Personalpronomens (Hat der eine Adresse?).

Auf der semantischen Ebene gibt es außerdem umgangssprachliche Begriffe und Ausdrücke (herumhämmern; Kram; übel zugerichtet), idiomatische Ausdrücke (alles, was das Herz begehrt) und typische Ausdrücke der gesprochenen Sprache (Was macht der Scotch?).

Auf syntaktischer Ebene sind neben kurzen oder manchmal sehr kurzen Sätzen überwiegend parataktische Strukturen zu beobachten:

- Phänomene von Ellipsen ([Ist es alles] In Ordnung?), manchmal so umfangreich, dass die Ausdehnung der Äußerung ([Sollte Ihnen] Noch so ein Abenteuer[passieren]) auf einen einzigen Bestandteil (Kompliment; Feuer?) drastisch reduziert wird

- Wegfall von Subjektpronomen mit anschließender Verberststellung ([Es] Ist scheinbar ein anstrengender Job)

- Asyndetische Nebensätze (Geben Sie zu, Sie sind anständig demoliert)

- am + Infinitiv (progressiv) (Bin am überlegen, ob ich dich zu 'ner Runde einlade)

- Weglassung des Artikels ([die] Bushaltestelle ist übrigens um die Ecke)

- Gebrauch von Demonstrativpronomen statt Personalpronomen (Hat der eine Adresse?).

Besonders häufig sind markierte Strukturen wie zum Beispiel:

- Topikalisierung von rhematischen Elementen ('n Schluck Bier oder 'n belegtes Brötchen gibt's hier nicht?)

- Linksversetzung (Die Freundin, woher hat sie das?)

- Rechtsversetzung (Hat mich angeschwindelt, der Arsch)

\footnotetext{
6 Aus Gründen der Lesbarkeit wird in diesem Paragraphen auf die genaue Angabe der Seite verzichtet, da es sich hierbei meist um wiederkehrende Ausdrücke handelt.
} 
- Nachträge (Mach ich. Ab Übermorgen)

- Deklarative Fragen (Ahmed hat deutsch mit ihm gesprochen?)

Schließlich gibt es auf der dialogischen Ebene zahlreiche Anredeformeln, die je nach Situation in einem neutralen, freundlichen oder ironisch-sarkastischen Ton ausgesprochen werden (Was haben Sie denn so tortenmäßig anzubieten, Verehrte?; Bis demnächst, Entlein; Okay, starker Mann) und Gesprächspartikeln, letztere oft an die erste Stelle gesetzt, um die Einstellung des Sprechers zu seinem Gesprächspartner zu signalisieren (Tja, [...]; Mhm, [...]; Ach Gott, [...]; Na, [...]; Na, Na dann, [...]). Auf dieser Ebene können wir auch Wiederholungen, Reformulierungen (Chef gefunden? - Chef gefunden; Waren Sie an dem Tag zufällig zu Hause? - Ja, war ich) und die Co-Konstruktion von syntaktischen Strukturen über mehrere turns hinweg (Sie wollten nicht, daß ich... - Daß Sie zu mir gehen?).

\section{6 Übersetzungsstrategien in der italienischen Version}

Hier halten wir zunächst fest, dass am besten die diastratische Variation in der Übersetzung erhalten ist, da sie sich hauptsächlich auf die semantisch-lexikalische Ebene konzentriert. In diesem Bereich ist die Übersetzung in der Lage, die soziolinguistische Markierung des Ausgangstextes überzeugend zu übertragen, auch dank der relativ großen Übereinstimmung der beiden Sprachen in Bezug auf das jeweilige Slang-Repertoire, wie im Beispiel (2) und (3): ${ }^{7}$

(2) Is möglich, dass sie genauso an der Fixe hängt wie du (Arjouni 1985/2003: 46)

(2') È possibile che si faccia anche lei (Arjouni/Maneri 1993/2009: 64)

(3) Fixern die Fixe zu bezahlen, ist kein Anlaß zu Luftsprüngen (Arjouni 1985/2003: 47)

(3') Non è che pagare le pere ai tossici mi faccia fare salti di gioia (Arjouni/Maneri 1993/2009: 65)

Die diatopische Variation hingegen ist erwartungsgemäß diejenige, die in der Übersetzung am stärksten neutralisiert wird. Es wird jedoch versucht, die Verwendung des Dialekts durch seine Übertragung auf die diaphasische Ebene zu erhalten, was sich unter anderem in der Verwendung von morphosyntaktischen Merkmalen widerspiegelt, die für ein „italiano dell'uso medio“ typisch sind, mit einem daraus resultierenden Unterschied in Bezug auf die diastratische Markierung. Während der Dialekt im Roman einer niedrigen Diastratie entspricht, weist die Übersetzung keinen vergleichbaren soziolinguistischen Charakter auf:

(4) Uff Hans! Dringe merr noch aan! (Arjouni 1985/2003: 26)

(4') Uffa, Hans, facciamocene ancora uno! (Arjouni/Maneri 1993/2009: 37)

(5) Isch habb kei Kaffe, des tut mer leid. Die Leut wolle ja aach nie Kaffe, die wolle immer nur Bier, aach so frie morjens, egelhaft (Arjouni 1985/2003: 70)

(5') Niente caffè, mi dispiace. La gente qui non chiede mai caffè, quelli vogliono solo birra, anche al mattino presto, uno schifo (Arjouni/Maneri 1993/2009: 95)

Was schließlich die diaphasische Variation betrifft, so erscheint sie insgesamt schwächer als im Original, trotz der unbestreitbaren Bemühungen der Übersetzerin, den umgangssprachlichen Stil in den Dialogen und in der Erzählerstimme mit den für diesen Zweck im Zieltext üblichen

\footnotetext{
${ }^{7}$ Die Hervorhebungen wurden von der Autorin des vorliegenden Beitrages vorgenommen, um auf die jeweiligen Besonderheiten zwischen ausgangsprachlichem und zielsprachlichem Text hinzuweisen.
} 
Mitteln wiederzugeben. Der Verlust der diaphasischen Markierung in den Dialogen ist in erster Linie darauf zurückzuführen, dass im Ausgangstext zahlreiche phonemisch-graphemische Indikatoren für Mündlichkeit vorkommen. Weniger gerechtfertigt ist der stilistische Unterschied auf der diegetischen Ebene, der nur geringfügig von Phänomenen auf dieser Ebene der Variation gekennzeichnet ist. Was das soziolinguistische Profil des Zieltextes in dieser Dimension der Variation weniger deutlich macht, sind vor allem zwei Phänomene, die sowohl die morphosyntaktische Ebene als auch die semantisch-lexikalische Ebene betreffen: Das erste Phänomen betrifft die zahlreichen Fälle von stilistischer Verschiebung (Normalisierung von markierten Strukturen), die sich vor allem in Dialogen zeigen. Hier finden sich normalisierende Lösungen auf der morphosyntaktischen Ebene, i. e. in Standardisierungen markierter Konstruktionen (6), im Gebrauch eines formelleren Registers (z. B. Konjunktiv statt Indikativ (7)), oder durch die Widergabe von Koordination durch den absoluten Ablativ (8):

(6) Adresse hat er? (Arjouni 1985/2003: 110)

(6') Ha un indirizzo? (Arjouni/Maneri 1993/2009: 146)

[Un indirizzo ce l'ha? ${ }^{8}$

(7) Hat's glaub ich in der Zeitung gelesen (Arjouni 1985/2003: 60)

(7') Credo che l'abbia letto sul giornale (Arjouni/Maneri 1993/2009: 81)

[L'avrà letto sul giornale]

(8) Sie wieherten los. Die Pfannibäuche wabbelten (Arjouni 1985/2003: 17)

(8') Scoppiarono in una risata equina, le pancette ballonzolanti (Arjouni/Maneri 1993/2009: 26)

[[...] che gli fece ballonzolare le pancette]

Normalisierende Lösungen finden sich auch auf der lexikalischen Ebene, wie z. B. die Verwendung von Wörtern oder Ausdrücken, die typisch für ein formelleres Register sind:

(9) Bevor Löff noch mehr Unsinn loswerden konnte, schob ich mich kurzerhand vor ihn an die Theke. (Arjouni 1985/2003: 135)

(9') Prima che Löff potesse snocciolare altre sciocchezze, mi interposi senza troppi complimenti tra lui e il bancone (Arjouni/Maneri 1993/2009: 179)

[m'infilai]

Das zweite Phänomen hingegen besteht aus der Tendenz zur Explizierung, die sich auf syntaktischer Ebene im Sinne der Vervollständigung von elliptischen Sätzen (10) und Expansionen in erklärender oder emphatischer Funktion (11), auf semantisch-lexikalischer Ebene im Sinne von transparenteren Übersetzungen angesichts von mehrdeutigen Ausdrücken (12) zeigen:

(10) Ich zog ein Jackett über und betrachtete die Beule unter der Schulter im Spiegel. Wie ein Neger im Solarium. (Arjouni 1985/2003: 44)

(10') Mi misi una giacca e controllai allo specchio il rigonfiamento sotto la spalla. Passavo inosservato più o meno come un negro in un centro abbronzatura. (Arjouni/Maneri 1993/2009: 61)

(11) Ganz Polizeikommissar, kratzte er sich am Kopf. (Arjouni 1985/2003: 89)

\footnotetext{
${ }^{8}$ In eckigen Klammern wird jeweils die korrekte standardsprachliche Form angegeben.
} 
(11') Di nuovo nei panni del commissario di polizia si grattò la testa. (Arjouni/Maneri 1993/2009: 119)

[wörtlich : Commissario di polizia fino al midollo]

(12) [sie] suchte nervös in einem Stück Krokodil. (Arjouni 1985/2003: 62)

[wörtlich: in un pezzo di coccodrillo]

(12') frugò nervosa in una borsetta di coccodrillo. (Arjouni/Maneri 1993/2009: 84)

$\mathrm{Zu}$ diesen beiden Phänomenen kommt aber noch ein drittes Phänomen, das die Tendenz betrifft, die diaphasische Markierung, die im ZT auf phonetischer und graphematischer Ebene ausgedrückt ist, auf die lexikalische und syntaktische Ebene zu verlagern:

(13) Hasse auch noch 'ne Kippe? (Arjouni 1985/2003: 46)

(13') Hai anche una svapora? (Arjouni/Maneri 1993/2009: 66)

Ausgangstext (AT): lexikalische Ebene (Kippe) und phonetisch-graphematische Ebene (Hasse; 'ne)

Zieltext (ZT): lexikalische Ebene (svapora)

(14) hass nich mal 'ne Maak für mich? (Arjouni 1985/2003: 45)

(14') ce l'hai un marco? (Arjouni/Maneri 1993/2009: 65)

AT: syntaktische Ebene (Auslassung des Pronomens) und phonetisch-graphematische Ebene (hass; nich; 'ne; Maak)

ZT: syntaktische Ebene (Rechtsversetzung)

Neben den illustrierten genannten Nivellierungen finden sich auch zahlreiche Kompensationsstrategien, z. B. Strategien wie die Verwendung von markierten Strukturen auf der morphosyntaktischen Ebene:

(15) Jammer nicht rum, hab mein Geld auch nicht auf der Straße gefunden. (Arjouni 1985/2003: 46)

(15') Piantala di piagnucolare, dopotutto io i soldi non li trovo per strada. (Arjouni/Maneri 1993/2009: 66)

AT: phonetisch-graphematische Ebene (rumjammern; hab)

ZT: syntaktische Ebene (Linksversetzung)

(16) Moment, ich muß den Kram auch aufschreiben. (Arjouni 1985/2003: 108)

(16') Un momento, tutta questa roba la devo anche scrivere. (Arjouni/Maneri 1993/2009: 144)

AT: lexikalische Ebene (Kram)

ZT: lexikalische Ebene (tutta questa roba) + syntaktische Ebene (Linksversetzung)

Auf der semantisch-lexikalischen Ebene findet sich die Verwendung einer Vielzahl von Formen - funktional zur Vermittlung von Expressivität, wie Phraseologismen, Vulgarismen, umgangssprachliche und Slangsausdrücke:

(17) Eine Dirne hatte irgendwann einmal nach Ahmed Hamul geschrien. (Arjouni 1985/2003: 43)

(wörtlich : aveva urlato il nome di ...)

(17') Una volta una prostituta era andata a cercare Ahmed Hamul alla stazione facendo una piazzata. (Arjouni/Maneri 1993/2009: 60) 
(18) Ich brauche ein paar Schriftstücke. (Arjouni 1985/2003: 90)

(wörtlich: 'documenti')

(18’) Ho bisogno di un paio di scartoffie. (Arjouni/Maneri 1993/2009: 121)

(19) Ich kam in Fahrt. (Arjouni 1985/2003: 41)

(wörtlich: 'mi sono messo in marcia'; sinngemäß: 'montai su tutte le furie')

(19’) Mi incazzai. (Arjouni/Maneri 1993/2009: 56)

Neben diesen Formen finden sich auch noch Anglizismen, pronominale Verben (ci facciamo) und die Augmentation:

(20) Während ich noch dabei war, mir meine Parade auszudenken. (Arjouni 1985/2003: 53)

(20’) Stavo ancora cercando di immaginarmi lo show. (Arjouni/Maneri 1993/2009: 72)

(21) [ich] spiele ein paar Partien Backgammon mit ihm. (Arjouni 1985/2003: 5)

(21') ci facciamo un paio di partite a backgammon. (Arjouni/Maneri 1993/2009: 12)

(22) Es war mühselig. (Arjouni 1985/2003: 105)

(22') Era un lavoraccio. (Arjouni/Maneri 1993/2009: 139)

In Bezug auf die Indikatoren der diaphasischen Markierung gibt es schließlich ein hohes Maß an soziolinguistischer Äquivalenz, dank der Verwendung von Merkmalen (Diskursmarker, Anreden etc.) mit einem gewissen Grad an soziolinguistischer Markierung in der Zielsprache, die mit den im AT verwendeten Formen vergleichbar sind.

\section{$7 \quad$ Schluss}

Wir haben uns am Anfang die Frage gestellt, ob die in der Praxis beobachtete Übersetzungsauswahl auch in unserem Korpus vorkommt und die Antwort ist - im Hinblick auf das untersuchte Werk - positiv.

Die in der italienischen Übersetzung von Happy birthday, Türke benutzten Strategien bestätigen die beobachteten Tendenzen in der aktuellen Übersetzungspraxis. Hierzu zählen:

- die Tendenz zur Standardisierung bzw. Einebnung der Sprachvariation (,law of growing standardization“, Toury 1995)

- die Tendenz zur Verlagerung der diatopischen Markierung - grundsätzlich unübertragbar (cf. Berruto 2010) - auf die diaphasische und diastratische Dimension (cf. Albrecht 2005)

- die Tendenz zur Reduzierung der funktionalen Phänomene zur Nachahmung des Gesprochenen bzw. deren Konzentration auf die lexikalische Ebene (cf. Schreiber 2007; Schwitalla/Tiittula 2009).

Durch eine Erweiterung der Datengrundlage und anhand der im Korpus vom Forschungsprojekt TRADIVARIO zu untersuchenden Texte wollen wir in Zukunft die Bandbreite der Strategien zur Wiedergabe von soziogeographischer Variation vergrößern und systematisieren, um Arbeitsgrundlagen für die universitäre Übersetzungsdidaktik zu entwickeln.

\section{Literaturverzeichnis}

Albrecht, Jörn (2005): Übersetzung und Linguistik. Tübingen: Narr.

Arjouni, Jakob (1985/2003): Happy Birthday, Türke! Zürich: Diogenes. 
Arjouni/Maneri (1993/2009): Happy birthday, turco! Italienische Übersetzung von Gina Maneri. Milano: Marcos y Marcos.

Assman, David-Christopher/Menzel, Nicola (2018): Textgerede. Interferenzen von Mündlichkeit und Schriftlichkeit in der Gegenwartsliteratur. München: Fink.

Baker, Mona (1996): „Corpus-based Translation Studies: the Challenges that Lie Ahead“. In: Somers, Harold (ed.): Terminology, LSP and Translation. Studies in Language Engineering in Honour of Juan C. Sager. Amsterdam/Philadelphia, Benjamins: 175-186.

Berruto, Gaetano (2010): Trasporre l'intraducibile: il sociolinguista e la traduzione. In: Sertoli, Giovanni/Vaglio Marengo, Carla/Lombardi, Chiara (eds.): Comparatistica e intertestualità. Alessandria, Edizioni dell'Orso: 899-910.

Betten, Anne (1994): Analyse literarischer Dialoge. In: Fritz, Gerd/Hundsnurscher, Franz (eds.): Handbuch der Dialoganalyse. Tübingen, Niemeyer: 519-544.

Calaresu, Emilia Maria (2005): Quando lo scritto si finge parlato. La pressione del parlato sullo scritto e i generi scritti più esposti: il caso della narrativa. In: Hölker, Klaus/Maaß, Christiane (eds.): Aspetti dell'italiano parlato. Münster, LiT: 65-92.

Cardinaletti, Anna/Garzone, Giuliana (eds.) (2005): L'italiano delle traduzioni. Milano: FrancoAngeli.

Cinato, Lucia/Amico di Meane, Isabella (2019): „TRADIVARIO. Variazione socio-geografica e traduzione: pratiche, strategie e tendenze nella coppia di lingue tedesco-italiano“. In: Calzoni, Raul/Moroni, Manuela (eds.): Passaggi, transiti e contatti tra lingue e culture: la traduzione e la germanistica italiana. Quaderni dell'AIG 2: 15-32.

Costa, Marcella (2015): Ritradurre Grass. La resa della variazione socio-geografica nelle traduzioni italiane di Die Blechtrommel. In: Costa, Marcella/Ulrich, Silvia (eds.): Riscritture e ritraduzioni. Intersezioni tra linguistica e letteratura tedesca. Alessandria, Edizioni dell'Orso: $75-88$.

Fallada, Hans (1933/2008): E adesso, pover'uomo? Übersetzung von Mario Rubino. Palermo: Sellerio. [Deutsche Erstveröffentlichung unter dem Titel Kleiner Mann, was nun? 1932. Rein beck bei Hamburg: Rowohlt].

Garzone, Giuliana/Cardinaletti, Anna (eds.) (2004): Lingua, mediazione linguistica e interferenza. Milano: FrancoAngeli.

Goetsch, Paul (1985): „Fingierte Mündlichkeit in der Erzählkunst entwickelter Schriftkulturen“. Poetica 17: 202-218.

Grass, Günter (1959/2007): Die Blechtrommel. Göttingen: Steidl.

Grass, Günter (1962/1989): Il tamburo di latta. Übersetzung von Lia Secci. Milano: Feltrinelli. Grass, Günter (2009): Il tamburo di latta. Übersetzung von Bruna Bianchi. Milano: Feltrinelli. Koch, Peter/Oesterreicher, Wulf (1985): „Sprache der Nähe - Sprache der Distanz“. Romanistisches Jahrbuch 36: 15-43.

Nicklaus, Martina (2015): Fingierte Mündlichkeit in literarischer Übersetzung. Die Rolle der Informationsstruktur. In: Gagliardi, Nicoletta (ed.): Die deutsche Sprache im Gespräch und in simulierter Mündlichkeit. Hohengehren, Schneider: 195-211.

Ondelli, Stefano/Viale, Matteo (2010): „L'assetto dell'italiano delle traduzioni in un corpus giornalistico. Aspetti qualitativi e quantitativi“. Rivista internazionale di tecnica della traduzione = International Journal of Translation 12: 1-62. openstarts.units.it/bitstream /10077/8159/1/Ondelli_Viale_RITT12.pdf [12.05.2021]. 
Schreiber, Michael (2007): Varietätenlinguistische Probleme der Übersetzung (romanischdeutsch). In: Di Meola, Claudio (ed.): Perspektiven Zwei: Akten der 2. Tagung Deutsche Sprachwissenschaft in Italien (Rom, 9.-11. Februar 2006). Roma, Istituto Italiano di Studi Germanici: 459-469.

Schwitalla, Johannes/Tiittula, Liisa (2009): Mündlichkeit in literarischen Erzählungen. Sprachund Dialoggestaltung in modernen deutschen und finnischen Romanen und deren Übersetzungen. Tübingen: Stauffenburg-Verlag.

Toury, Gideon (1995): Descriptive translation studies and beyond. Amsterdam/Philadelphia: Benjamins. 